Michael Kurin, MD

Digestive Health Institute, University Hospitals

Cleveland Medical Center; Case Western

Reserve University School of Medicine,

Cleveland, $\mathrm{OH}$
Gregory Cooper, MD

Digestive Health Institute, University Hospitals Cleveland Medical Center; Director of Gastroenterology and Hepatology Fellowship, University Hospitals Cleveland Medical Center; Professor, Department of Medicine, and Professor, Department of Population and Quantitative Health Sciences, Case Western Reserve University School of Medicine, Cleveland, $\mathrm{OH}$

\title{
Irritable bowel syndrome with diarrhea: Treatment is a work in progress
}

\section{ABSTRACT}

Irritable bowel syndrome (IBS) is a heterogeneous functional disease with a high prevalence and significant impact on quality of life. Traditionally understood as a pure disorder of brain-gut interaction, it is increasingly clear that IBS encompasses diverse pathologies, some of which involve objective alterations of intestinal structure, function, and the microbiome. IBS is subclassified as diarrhea, constipation, or mixed type based on the most prominent stool form. We review the diagnosis and management of the diarrheal type through a pathophysiologic lens, with attention to recent developments that can inform a mechanistically based targeted approach to treatment.

\section{KEY POINTS}

IBS is classified as IBS-diarrhea when at least $25 \%$ of bowel movements on symptomatic days are type 6 or 7 on the Bristol Stool Scale.

New research suggests that IBS has diverse pathologies that include intestinal inflammation, postinfectious sequelae that increase intestinal permeability, food sensitivities, microbiome alterations, and bile acid malabsorption.

Therapies are increasingly being targeted at one or more of these pathologies, leading to the availability of new treatments such as probiotics, bile acid sequestrants, and the low-FODMAP (fermentable oligosaccharides, disaccharides, monosaccharides, and polyols) diet.

First-line therapies still include antidiarrheals, regular exercise, psychological therapy, and the traditional IBS diet.

doi:10.3949/ccjm.87a.19011
T RRITABLE BOWEL SYNDROME (IBS) remains a clinical diagnosis, and its treatment is still mostly empiric and focused on relieving symptoms. That said, our understanding of its mechanisms is progressing, and treatments are increasingly targeted to the etiology in the individual patient.

\section{A FUNCTIONAL DISEASE}

IBS is a functional disease characterized by chronic intermittent abdominal pain and altered bowel habits. ${ }^{1}$ Patients may also experience postprandial or stress-related abdominal bloating and sensation of incomplete emptying. ${ }^{2}$ Comorbid dyspepsia, mood disorder, chronic migraines, interstitial cystitis, and fibromyalgia are common. ${ }^{2}$

The estimated national prevalence is $10 \%$ to $12 \%,{ }^{3}$ although some estimates are as high as $21 \% .{ }^{1}$ There is a well-documented $3: 1 \mathrm{fe}$ male predominance. ${ }^{2}$ This disorder accounts for $25 \%$ to $50 \%$ of all gastroenterology referrals nationwide, and its healthcare burden exceeds $\$ 20$ billion annually. ${ }^{4}$

Irritable bowel syndrome has 3 subtypes:

IBS-diarrhea (IBS-D) is diagnosed when at least $25 \%$ of bowel movements on symptomatic days are type 6 (mushy consistency without clear edges) or type 7 (completely liquid without solid substance) on the Bristol Stool Scale ${ }^{5,6}$

IBS-constipation (IBS-C) is diagnosed when $25 \%$ of bowel movements are type 1 (hard and lumpy) or type 2 (sausage-like and lumpy).

IBS-mixed (IBS-M) is diagnosed when both criteria are fulfilled.

About one-third of patients fall into each subtype. ${ }^{3}$ This review focuses on the diagnosis and management of IBS-D. 


\section{TABLE 1}

\section{Diagnoses to consider before irritable bowel syndrome-diarrhea}

Inflammatory bowel disease

Food intolerance or sensitivity

Small intestinal bacterial overgrowth

Bile acid diarrhea

Pancreatic exocrine deficiency

Medication side effects

Functional diarrhea

Colon cancer

Chronic parasitic infection

Microscopic colitis

Thyroid disease

Celiac disease

\section{TABLE 2}

\section{Alarm signs and symptoms}

Age over 50

Gastrointestinal bleeding

Anemia

Fever

Night sweats

Unintentional weight loss

Family history of organic gastrointestinal disease

Other symptoms that should alert provider to consider another diagnosis

Nocturnal symptoms

Symptoms that persist when fasting

Low fecal osmotic gap (fecal osmotic gap $=290$ $\mathrm{mOsm} / \mathrm{kg})-2 \times($ stool $\mathrm{Na}+$ stool K); a low gap $(<50 \mathrm{~m} 0 \mathrm{sm} / \mathrm{kg}$ ) suggests a secretory cause of diarrhea such as microscopic colitis. Patients with IBS would be expected to have a normal gap.

\section{DIAGNOSIS}

Endoscopy is not recommended in patients who meet Rome IV criteria unless they have alarm signs or laboratory abnormalities
The most widely accepted set of diagnostic criteria for IBS is Rome IV, ie, recurrent abdominal pain at least 1 day per week in the last 3 months that is (at least 2 of the following required):

- Related to defecation

- Associated with a change in stool frequency

- Associated with a change in stool form.

A validation study of the Rome IV criteria was performed at 9 sites in 3 countries and showed a $62 \%$ sensitivity and $97 \%$ specificity, although patients with inflammatory bowel disease, celiac disease, and diabetes were excluded. ${ }^{7}$ The gold standard was normal findings on endoscopy and a physician diagnosis of IBS, which carries inherent subjectivity that detracts from the veracity of these statistics. The Rome IV criteria are not able to differentiate IBS from other causes of lower gastrointestinal symptoms, especially those not visible on endoscopy.

When evaluating patients who meet Rome IV criteria, many other disorders must be considered (Table 1). We also advise against limiting IBS diagnosis to patients with abdominal "pain." All therapies approved by the US
Food and Drug Administration for IBS have been studied on the basis of earlier Rome criteria, which also included patients with abdominal "discomfort."

In the past, the exclusion of other causes of IBS symptoms centered around endoscopic workup to exclude inflammatory bowel disease. Now, endoscopy is not recommended in patients who meet Rome IV criteria unless they have alarm signs (Table 2) or laboratory abnormalities because the odds of finding inflammatory bowel disease, celiac disease, colon cancer, or microscopic colitis in this setting are negligible (Figure 1). ${ }^{3}$

In the absence of alarm signs, laboratory tests such as fecal calprotectin (reference range $<40 \mu \mathrm{g} / \mathrm{g}$ ) are considered sufficient to effectively exclude inflammatory bowel disease. ${ }^{1,3}$ Alternatively, some recommend serum C-reactive protein $(<0.5 \mathrm{mg} / \mathrm{dL})^{3}$ and fecal lactoferrin $(<7 \mu \mathrm{g} / \mathrm{g}){ }^{8}{ }^{8}$

Routine screening for celiac disease is recommended by some guidelines, 3,8 based on a meta-analysis that reported a nearly 10 -fold higher prevalence than in the general population. ${ }^{9}$ However, a more recent observational study showed a $0.41 \%$ prevalence in both IBS- 


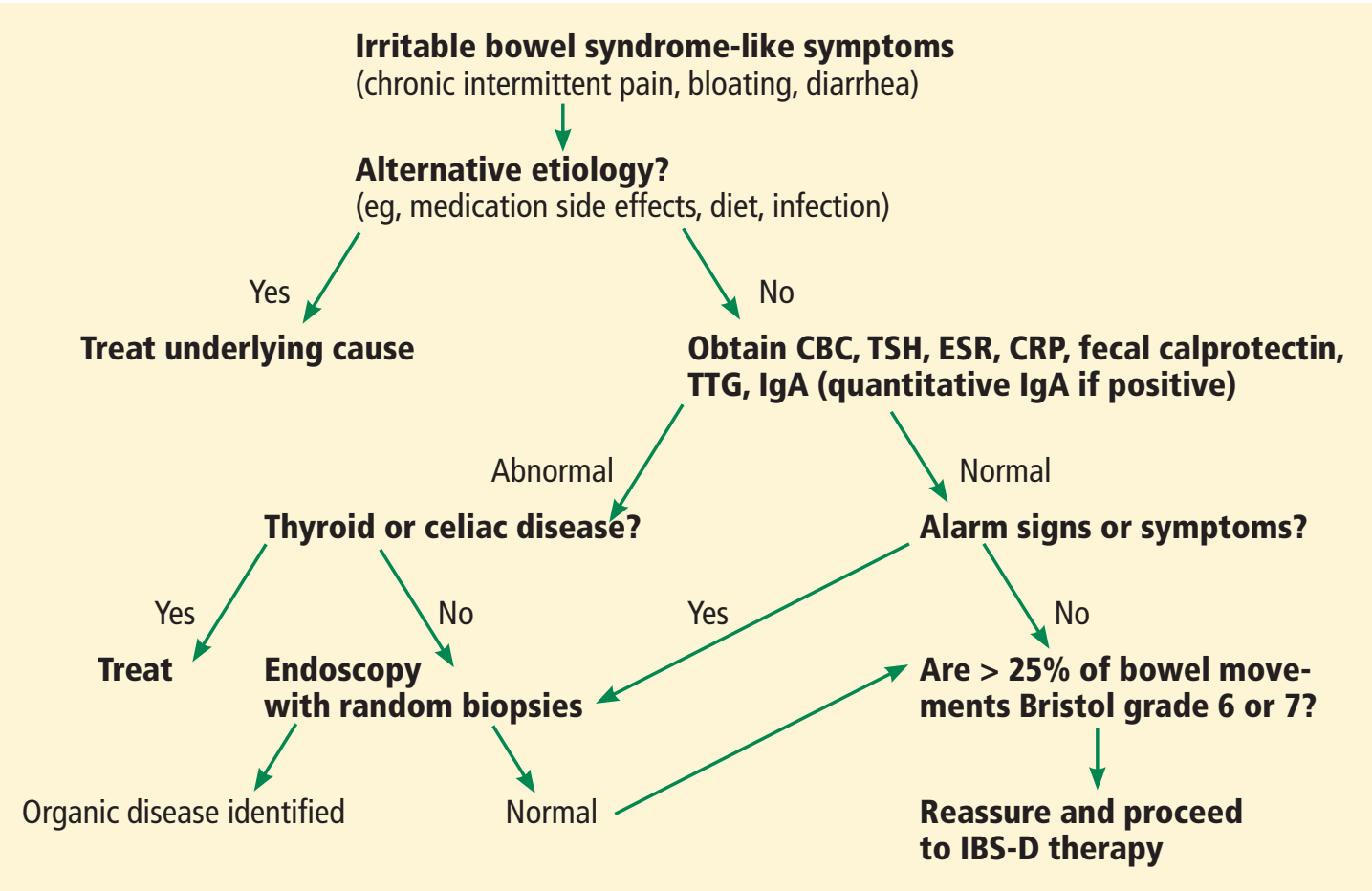

$\mathrm{CBC}=$ complete blood cell count; $\mathrm{CRP}=\mathrm{C}$-reactive protein; $\mathrm{ESR}=$ erythrocyte sedimentation rate; $\lg \mathrm{A}=$ immunoglobulin $\mathrm{A}$;

TSH = thyroid-stimulating hormone; TTG = tissue transglutaminase

Figure 1. Algorithm for diagnosing irritable bowel syndrome-diarrhea (IBS-D).

$\mathrm{D}$ and the general population. ${ }^{10}$

Any patient with suspected IBS undergoing screening colonoscopy should have random biopsies of the right and left colon to rule out microscopic colitis. ${ }^{1}$

\section{MANAGEMENT}

Management of IBS-D should be targeted to its underlying etiology. However, in the absence of a clear understanding of the mechanisms that produce symptoms, treatments have traditionally focused on symptom relief, namely, antidiarrheals.

\section{Antidiarrheal therapy}

Loperamide, the best studied antidiarrheal, is a synthetic opioid that slows intestinal motility and increases absorption of water and electrolytes, leading to firmer and less frequent stools. ${ }^{11}$ In several older studies reinforced by a meta-analysis, loperamide improved diarrhea in patients with IBS-D, but it had little effect on other symptoms such as abdominal pain. ${ }^{11}$
Many clinicians choose loperamide as a firstline therapy for IBS-D due to availability, low cost, and minimal adverse effects at low doses.

Soluble fiber supplements such as psyllium that act as stool bulking agents are recommended by recent guidelines for the management of IBS. ${ }^{3}$ Although their use in IBS-D may be counterintuitive, these supplements may improve stool consistency. However, their use in IBS-D specifically has not been adequately studied.

\section{Therapy targeting the brain-gut axis}

Traditionally, IBS has been understood as a disorder of brain-gut interactions manifesting as visceral hypersensitivity. ${ }^{12}$ Patients may experience an exaggerated sensory response to intestinal contractions, distention, and perhaps microinflammation ${ }^{13}$ due to sensitization of afferent nerves in the gut wall, pre- or postganglionic efferent nerves, or central nerves. ${ }^{12}$

Central nerves, perhaps stimulated by psychosocial stressors, may also contribute to ir-
Many clinicians choose loperamide as a first-line therapy for IBS-D 
regular peristalsis. ${ }^{12}$ Although studies have not identified a consistent pattern of disordered gastrointestinal peristalsis, ${ }^{12}$ a subset of IBS patients have a higher frequency of powerful colonic contractions, called high-amplitude propagating contractions, which typically lead to cramping and urgency. ${ }^{13,14}$

Therapies targeted toward this view of IBS have been used for decades.

Cognitive behavioral therapy is recommended as first-line ${ }^{4}$ or second-line therapy. ${ }^{15}$ In a large meta-analysis, clinic-administered cognitive behavioral therapy decreased the risk of persisting symptoms compared with placebo (hazard ratio [HR] 0.60). ${ }^{15}$

The benefit of self-administered cognitive behavioral therapy (ie, the patients learn how to perform the techniques on themselves) is less clear. A recent meta-analysis found no significant benefit, although some individual studies have shown that it was useful. ${ }^{16,17}$ Efficacy may depend on how this therapy is administered.

A recent meta-analysis reported significant benefit of relaxation therapy (number needed to treat $[\mathrm{NNT}]=6$ ), multicomponent psychological therapy $(\mathrm{NNT}=4)$, hypnother-

Peppermint oil is an underappreciated treatment of IBS apy $(\mathrm{NNT}=5)$, and dynamic psychotherapy $(\mathrm{NNT}=4) .{ }^{17}$ Mindfulness meditation, stress management, and cognitive behavioral therapy administered via the internet were not found to significantly reduce the risk of persistent symptoms. The authors noted that all psychological therapies delivered without personal contact between the patient and therapist lacked significant benefit.

Tricyclic antidepressants can modulate pain and slow gastrointestinal motility through their anticholinergic effects. ${ }^{17}$ In a large, recently updated meta-analysis that included a variety of tricyclic antidepressants, the risk of persistent IBS symptoms was lower than that with placebo (HR 0.65, NNT = 4.5). ${ }^{17}$ The authors did not suggest a preference for any particular agent of this class.

Selective serotonin reuptake inhibitors can increase gastrointestinal motility via serotonin receptors of the enteric nervous system. Their use should be reserved for constipationtype IBS, although most trials of antidepressant therapy did not differentiate between IBS subtypes. ${ }^{17}$ There have been no randomized clinical trials of serotonin-norepinephrine reuptake inhibitors for IBS. ${ }^{17}$

Antispasmodics are thought to decrease symptoms of pain by relaxing gut contractions and slowing motility. ${ }^{18}$ They are intended for short-term use, after meals. Their use is limited by anticholinergic side effects, including constipation, but guidelines recommend their use. ${ }^{3}$

A recent meta-analysis of antispasmodic use showed significant improvement in overall IBS symptoms $(\mathrm{NNT}=5) \cdot{ }^{3}$ Specifically, otilonium $(\mathrm{NNT}=5)$, pinaverium $(\mathrm{NNT}=$ 4), hyoscine butylbromide (NNT = 3), dicyclomine $(\mathrm{NNT}=4)$, and drotaverine (NNT $=2$ ) were all found to significantly improve overall symptoms. ${ }^{3}$ The overall quality of the data, however, is limited by the age of the trials, with nearly all having occurred 2 to 3 decades ago, with the exception of drotaverine and pinaverium.

Combination therapy with the antispasmodic mebeverine and cognitive behavioral therapy was more effective than mebeverine alone after 3 months in a randomized controlled trial $(\mathrm{NNT}=5) \cdot{ }^{19}$ However, after 12 months, there was no longer a statistically significant difference between the 2 groups.

Melatonin has been studied as a potential therapy for IBS, given its involvement in the regulation of gastrointestinal motility, nociception, and possible anti-inflammatory properties. ${ }^{20}$ Melatonin has been shown to reduce abdominal pain in patients with IBS, but its suitability for IBS-D patients in particular has not yet been studied. ${ }^{20}$ It is not among the therapies for IBS-D endorsed by a published guideline.

Peppermint oil is an underappreciated treatment of IBS. It has antispasmodic and anti-inflammatory properties and serotonin $5-\mathrm{HT}_{3}$ receptor antagonism that can slow motility and may decrease visceral hypersensitivity. ${ }^{21}$ It is used as first-line therapy in Europe due to its minimal adverse side effect profile. ${ }^{21}$ A recent meta-analysis of peppermint oil use showed that it significantly improved overall symptoms $(\mathrm{NNT}=4){ }^{3}$

Care must be taken in prescribing peppermint oil to patients with gastroesophageal reflux disease, however, because peppermint relaxes the gastroesophageal sphincter. This side effect can be limited with a delayed-release 
form that has recently been shown to induce a $67 \%$ reduction in severe symptoms after 4 weeks of use compared with $35 \%$ with placebo $(\mathrm{NNT}=3.0) .^{21}$

\section{Serotonin receptor antagonism}

Serotonin is an important neurotransmitter in the gut that plays a prominent role in inducing peristalsis, intestinal distention, and contraction and modulating sensation of intestinal stimuli both centrally and peripherally. ${ }^{22}$ Patients with IBS-D have been shown to have high postprandial serum levels of serotonin. ${ }^{23,24}$

Alosetron and ondansetron are serotonin 5- $\mathrm{HT}_{3}$ receptor antagonists that decrease gastrointestinal motility and may modulate pain perception. ${ }^{24}$

Alosetron is a potent and effective therapy for IBS-D, with meta-analysis data showing significant benefit $(\mathrm{NNT}=7),{ }^{25}$ but it was withdrawn from the market in 2001 due to reports of ischemic colitis. ${ }^{26}$ However, it has recently been reinstituted for compassionate use at a lower dose (0.5 mg twice a day).

Ondansetron is less potent than alosetron, but a recent placebo-controlled randomized crossover study ${ }^{27}$ showed that, compared with placebo, it reduced the frequency of stools by $11 \%$, reduced bloating and urgency by 1 day per week, and decreased gut transit time by 10 hours, although it did not decrease abdominal pain, and it had a minimal adverse effect profile. ${ }^{27}$ The mean dose of ondansetron was $4 \mathrm{mg}$ daily.

\section{An opioid agonist and antagonist}

Eluxadoline is a mu- and kappa-opioid agonist and a delta opioid antagonist. It is thought to regulate gastrointestinal motility, intestinal secretion, and visceral sensation and provide central analgesia. ${ }^{28}$

In pooled data analysis of 2 recent randomized controlled trials involving 2,427 patients, those using eluxadoline had decreased abdominal pain and improvement in stool consistency compared with placebo $(\mathrm{NNT}=$ 9). ${ }^{28}$ Adverse effects include sphincter of Oddi spasms $(0.5 \%)$ in patients with previous cholecystectomy, including some that manifested as pancreatitis. ${ }^{29}$ This led to a US Food and Drug Administration warning against use of eluxadoline in patients without a gallblad- der. ${ }^{30}$ The modest degree of benefit, along with the safety profile and cost of eluxadoline, explains why some gastroenterologists prefer other available therapies.

\section{THERAPY TARGETING AN UNDERLYING INTESTINAL ABNORMALITY}

Recent developments have suggested novel disease mechanisms that have diversified our understanding of IBS. Five emerging theories of increasing relevance are intestinal inflammation, postinfection, food sensitivity, microbiome alterations, and bile acid malabsorption. ${ }^{1,2}$

\section{Intestinal inflammation}

Patients with IBS may have a subtle but abnormal increase in inflammatory cells in the bowel, especially in close proximity to nerves. ${ }^{31}$ An increased number of activated mast cells and heightened cytokine production caused by release of serine proteases is one suggested mechanism. ${ }^{1,2}$ It is possible that eosinophils also play a role, as they have recently been found in large numbers in the intestines of patients with nonceliac gluten sensitivity, a condition that considerably overlaps with IBS in clinical presentation. ${ }^{32}$

Based on this theory, a variety of anti-inflammatory therapies has been used in trials for IBS, mostly with negative results.

Prednisolone in moderate daily doses was compared with placebo in postinfectious IBS-D. ${ }^{33}$ It lacked benefit, although patients already taking steroids may be at lower risk of developing IBS. ${ }^{34}$

Several 5-aminosalicylic acids have been used in trials as well. A recent meta-analysis of mesalazine found no benefit compared with placebo. $^{31}$

Other anti-inflammatory agents. Encouragingly, though, several recent studies assessed therapies that reduce mast cell activation and its effects, including the mast cell stabilizers cromoglycate and ketotifen, the histamine-1 receptor antagonist ebastine, and the dietary supplements palmitoylethanolamide and polydatin with largely positive results. ${ }^{35-40}$

While promising, these therapies remain controversial, have not yet reached mainstream practice, and have not been endorsed by any major guidelines.
A variety of anti-inflammatory therapies have been used in trials for IBS, mostly with negative results 


\section{TABLE 3}

\section{Proposed diets for irritable bowel syndrome}

\section{Traditional IBS diet}

Eat small, frequent meals

Reduce gas-producing foods, including: Soda

Juice

Caffeine

Beans

Onions

Bagels

Pretzels

Alcohol

Wheat

Certain fruits

\section{Modified NICE diet}

Eat small, frequent meals

Limit high-fiber foods

Avoid:

Alcohol

Caffeine

Soda

Sorbitol

\section{Low-FODMAP diet}

Avoid wheat, selected fruits and vegetables, corn syrup, onions

FODMAP = fermentable oligosaccharides, disaccharides, monosaccharides, and polyols; NICE = National Institute for Health and Care Excellence

\section{Postinfectious pathophysiology}

During acute gastrointestinal infection, there is a transient increase of lymphocytes and neuroendocrine cells in the gastrointestinal tract. These can alter motility through serotonin production and increase intestinal permeability. It is postulated that these abnormalities occasionally persist, leading to IBS, ${ }^{1,2}$ as has recently been shown after Giardia infection. ${ }^{41}$

This theory is supported by the discovery that patients with IBS have elevated levels of 2 key antibodies in the bacteria-host interaction during acute gastrointestinal illness: anti$\mathrm{CdtB}$ and antivinculin antibodies. ${ }^{42}$ In fact, antivinculin antibodies themselves may play a role in the complicated postinfectious pathophysiology, as decreased levels of vinculin can lead to weaker cell-cell adhesion and decrease the integrity of the extracellular matrix, making the intestine more permeable. ${ }^{43}$ It may also alter gut motility by binding with actin located near the interstitial cells of Cajal, which help regulate motility. ${ }^{43}$

Glutamate. Until recently, there were no therapies targeted to this mechanism, but a recent small randomized controlled trial of glutamate, a dietary supplement purported to reduce intestinal permeability, showed markedly positive effects compared with placebo in postinfectious IBS $(\mathrm{NNT}<2) .{ }^{44}$ However, the reproducibility of these results has been questioned, ${ }^{45}$ and this therapy is not currently recommended by any guideline.

\section{Food sensitivity}

Most patients with IBS believe their symptoms are related to diet. ${ }^{3,4}$ Unlike with food allergy, there is no established way to identify specific food sensitivities. In one study, patients with IBS were placed on exclusion diets based on their serum immunoglobulin $G$ ( $\operatorname{lgG}$ ) titers to various food antigens and compared with a sham diet group. ${ }^{46}$ There was a significant decrease in symptom severity in the true diet group, especially when those who did not adhere to the diet were excluded $(\mathrm{NNT}=2.5)$.

While encouraging, most of the foods that were excluded were those already known to cause increased symptoms in IBS patients such as wheat, milk, and yeast, perhaps rendering IgG testing unnecessary. This method is not currently used to devise diets for IBS patients.

FODMAPs. Elimination of fermentable oligosaccharides, disaccharides, monosaccharides and polyols (FODMAPs) is recommended by the guidelines. ${ }^{1}$ FODMAPs are sugars that ferment in the gut due to inadequate digestion; common ones are lactose, fructose, fructans, and sorbitol. Foods containing FODMAPs include wheat, some fruits and vegetables, corn syrup, and onions.

An initial observational study showed significant symptom improvement in 74\% of IBS patients adhering to a low-FODMAP diet. ${ }^{47}$ However, this study only included patients with a positive fructose breath test and did not 
include a control group.

A subsequent small randomized controlled trial reported a mean $30 \%$ decrease in symptom severity with a low-FODMAP diet compared with a typical Australian diet. ${ }^{48}$ However, subsequent randomized controlled trials that compared the low-FODMAP diet with a traditional IBS diet, ${ }^{49}$ or the modified UK National Institute for Health and Care Excellence (NICE) diet (Table 3$)^{50}$ showed no difference in efficacy - each diet caused global symptom improvement in approximately 50\% of patients. The low-FODMAP diet led to decreased abdominal pain compared with the modified NICE diet. Therefore, while a lowFODMAP diet is recommended by the guidelines, ${ }^{3}$ it is also reasonable to prescribe other, simpler diets. Moreover, nonresponders at 4 weeks should discontinue the low-FODMAP diet and start an alternative one. Patients should be encouraged to eat small, healthy meals frequently and exercise. ${ }^{1}$

Conversely, patients who do improve with a low-FODMAP diet after 4 weeks must carefully reintroduce FODMAPs to devise a longterm individualized diet under the guidance of a trained nutritionist.

Lactose. Nearly $40 \%$ of IBS patients are estimated to have lactose maldigestion. ${ }^{2}$ Some experts recommend excluding lactose even in patients without true lactose intolerance. ${ }^{2}$

Gluten elimination is not recommended. ${ }^{3}$ While one randomized controlled trial appeared to show that a gluten-free diet was beneficial in patients with IBS, ${ }^{51}$ a subsequent trial showed no benefit in those already on a low-FODMAP diet. ${ }^{52,53}$ The earlier study did not differentiate between gluten and fructans, and the symptom improvement was likely related to elimination of the FODMAP fructans rather than gluten.

\section{Microbiome alterations}

Some patients with IBS have an altered microbiome composition. ${ }^{53}$ Whether this constitutes small intestinal bacterial overgrowth remains controversial.

Part of this controversy is due to a lack of a gold standard diagnostic test for small intestinal bacterial overgrowth itself. Intestinal aspirates are expensive, unreliable, and technically difficult to obtain. ${ }^{54}$ The reliabil- ity of breath testing is controversial because a variety of substrates can be used, and most tests lack uniform criteria for positivity. ${ }^{55} \mathrm{Ab}$ normal breath tests are common among IBS patients, ranging from $35 \%$ to $84 \% .{ }^{56}$

A meta-analysis using case-control studies of patients with IBS and healthy controls showed that the IBS patients were 3 times more likely to have an abnormal breath test. ${ }^{55}$ The glucose breath test appears to have the highest specificity for IBS. ${ }^{55}$ This has led some to hypothesize that some patients with IBS-like symptoms may truly have small intestinal bacterial overgrowth, or can be treated as such..$^{55}$

However, a study that applied the diagnostic criteria of an abnormal breath test and abnormal jejunal aspirate cultures to IBS patients compared with healthy controls found no difference between the 2 groups. ${ }^{56}$ When a lower cutoff for positivity of jejunal aspirates was used, IBS patients had a greater prevalence of positive criteria. This may suggest a milder form of overgrowth-like pathology.

Routine screening of IBS patients for small intestinal bacterial overgrowth is currently not standard practice, ${ }^{3}$ but research into targeted therapies that act on the microbiome is ongoing.

Low-FODMAP diet. Responders to the low-FODMAP diet experienced large changes in the composition of their microbiomes. ${ }^{57}$ Unfortunately, response to the diet was not predicted by baseline microbiome composition. There is hope that in the future, we will discover properties of patient fecal microbiota that may help predict which IBS patients will respond most to the low-FODMAP diet. ${ }^{57}$ Perhaps breath testing will prove effective at predicting response. ${ }^{47}$

Probiotics theoretically replenish the microbiome with certain bacteria, alter gut $\mathrm{pH}$, provide barrier protection and have antiinflammatory effects. ${ }^{31,58}$ Though the quality of data is poor and scattered among various formulations, probiotics are currently recommended for IBS-D, given their minimal risk. . $^{3,58}$

Rifaximin is an antibiotic that is poorly absorbed, which maximizes its effect on the gastrointestinal tract while minimizing systemic adverse effects. The Targeted, Nonsystemic Antibiotic Rifaximin Gut-Selective Evaluation of Treatment for IBS-D 1 (TAR-
Probiotics are currently recommended for IBS-D, given their minimal risk 


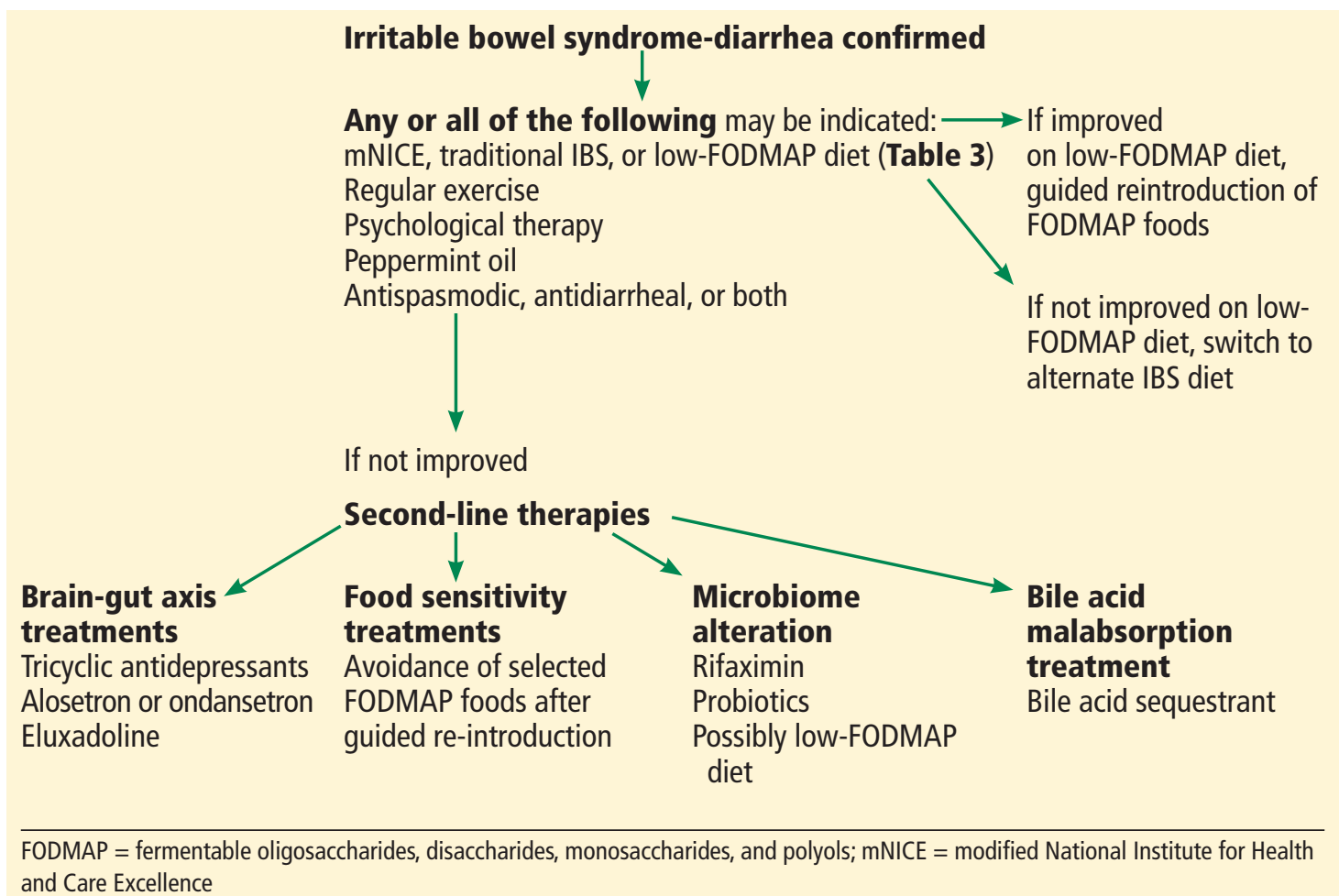

Figure 2. Suggested algorithm for treating irritable bowel syndrome-diarrheal type.

\section{Fecal microbiota transplant is not currently endorsed, but that may change}

GET 1) and TARGET 2 controlled trials showed greater adequate relief of IBS-D or IBS-M symptoms $(\mathrm{NNT}=10)$ in patients taking rifaximin compared with placebo with 3 months follow-up. ${ }^{59}$ The TARGET 3 study showed that retreatment with rifaximin remains effective if symptoms recur. ${ }^{60}$

While response to rifaximin correlates with improvement in lactulose breath testing, ${ }^{61}$ an attempt to predict response to rifaximin using lactulose breath testing was unsuccessful. ${ }^{61}$ Rifaximin is currently approved for empiric use in IBS-D. ${ }^{3}$

Fecal microbiota transplant. In a small randomized controlled trial, $65 \%$ of patients had significant improvement in their symptoms 3 months after receiving a fecal microbiota transplant (NNT $=4.5) .{ }^{62}$ Unfortunately, the benefit above placebo was not sustained at 12 months.

A subsequent meta-analysis showed no overall evidence of benefit from fecal microbiota transplant compared with placebo, although on subgroup analysis, patients who received only single-dose transplants had a modest benefit $(\mathrm{NNT}=5)$. A newly published randomized controlled trial showed a large benefit over placebo. ${ }^{63}$

Fecal microbiota transplant is not currently endorsed by any guideline for IBS-D, but that may change in light of the emerging evidence.

\section{Bile acid malabsorption}

Increased amounts of bile in the colon can increase colonic motility, fluid secretion, mucosal permeability, and visceral sensation. ${ }^{64}$ Bile acid malabsorption may be present in 30\% to $50 \%$ of IBS-D patients. ${ }^{1}$ Bile acid absorption can be detected by serum tauroselcholic acid (SeHCAT) level, fecal bile acid, serum 7 alpha-hydroxy-4-cholesten-3 (C4), or fibroblast growth factor 19 (FGF19) measurement.

In a recent study, abnormal SeHCAT levels in patients with IBS-D predicted response to treatment with a bile acid sequestrant with impressive accuracy. ${ }^{65}$ Unfortunately, SeHCAT testing is not currently available in the United States. Direct measurement of fecal bile acid requires 48-hour stool collection 
and a high-fat diet so it may be impractical for many patients.

Serum C4 and FGF19 were evaluated as biomarkers for bile acid malabsorption, both separately ${ }^{66,67}$ and more recently in combination, ${ }^{68}$ and have been found to have specificity and negative predictive value of approximately $80 \%$ but lower sensitivity and positive predictive value.

Abnormal serum C4 levels have previously been shown to predict response to bile acid sequestrant therapy in IBS-D, although in a very small sample. ${ }^{69}$ Based on this, some authors have recommended serum C4 and FGF19 as screening tests for bile acid malabsorption in IBS-D patients, ${ }^{68}$ but this has not reached mainstream practice and is not currently recommended by any guideline. Expert opinions are mixed regarding empiric use of bile acid sequestrants in IBS-D patients. ${ }^{8}$

\section{TARGETED THERAPY STILL A WORK IN PROGRESS}

Irritable bowel syndrome is a heterogeneous disease that is a conglomerate of several

\section{REFERENCES}

1. Chey WD, Kurlander J, Eswaran S. Irritable bowel syndrome: a clinical review. JAMA 2015; 313(9):949-958. doi:10.1001/jama.2015.0954

2. Mearin F, Lacy BE, Chang L, et al. Bowel disorders. Gastroenterology 2016; 150(6):1393-1407. doi:10.1053/j.gastro.2016.02.031

3. Ford AC, Moayyedi P, Chey WD, et al; ACG Task Force on Management of Irritable Bowel Syndrome. American College of Gastroenterology monograph on management of irritable bowel syndrome. Am J Gastroenterol 2018; 113(suppl 2):1-18. doi:10.1038/s41395-018-0084-x

4. American College of Gastroenterology Task Force on Irritable Bowel Syndrome; Brandt $\mathrm{L}$, Chey WD, Foxx-Orenstein, et al. An evidencebased position statement on the management of irritable bowel syndrome. Am J Gastroenterol 2009; 104(suppl 1):S1-S5. doi:10.1038/ajg.2008.122

5. Lewis SJ, Heaton KW. Stool form scale as a useful guide to intestinal transit time. Scand J Gastroenterol 1997; 32(9):920-924. doi:10.3109/00365529709011203

6. Drossman DA, Hasler WL. Rome IV-functional GI disorders: disorders of gut-brain interaction. Gastroenterology 2016; 150(6):1257-1261. doi:10.1053/j.gastro.2016.03.035

7. Palsson OS, Whitehead WE, van Tilburg MA, et al. Rome IV diagnostic questionnaires and tables for investigators and clinicians. Gastroenterology 2016; 150(6):1481-1491. doi:10.1053/j.gastro.2016.02.014

8. Smalley W, Falck-Ytter C, Carrasco-Labra A, Wani S, Lytvyn L, Falck-Ytter Y. AGA clinical practice guidelines on the laboratory evaluation of functional diarrhea and diarrhea-predominant irritable bowel syndrome in adults (IBS-D). Gastroenterology 2019; 157(3):851-854. doi:10.1053/j.gastro.2019.07.004

9. Ford AC, Chey WD, Talley NJ, Malhotra A, Spiegel BM, Moayyedi P. Yield of diagnostic tests for celiac disease in individuals with symptoms suggestive of irritable bowel syndrome: systematic review and meta-analysis. Arch Intern Med 2009; 169(7):651-658. pathologic mechanisms and degrees of severity that require individualized management strategies. Diagnosis remains clinical and extends beyond the Rome IV criteria. While the traditional understanding of IBS as a disorder of brain-gut interactions remains true, ongoing research has contributed to an evolving understanding of IBS that includes an increasing number of subtle yet objective microscopic intestinal abnormalities that likely contribute to the pathophysiology of IBS-D.

Therapies are increasingly targeting one or more of these mechanisms, leading to availability of several new treatment options (Figure 2). Identifying patients' precise mechanism of disease to enable targeted therapy remains a work in progress, but there is reason to hope this can be achieved in the near future. In the meantime, evidence-based therapy remains empiric, although clinicians are free to adjust the order in which approved therapies are attempted in accordance with their clinical suspicion for the most prominent symptoms or pathophysiology.

doi:10.1001/archinternmed.2009.22

10. Cash BD, Rubenstein JH, Young PE, et al. The prevalence of celiac disease among patients with nonconstipated irritable bowel syndrome is similar to controls. Gastroenterology 2011; 141(4):11871193. doi:10.1053/j.gastro.2011.06.084

11. Lesbros-Pantoflickova D, Michetti P, Fried M, Beglinger C, Blum AL. Meta-analysis: the treatment of irritable bowel syndrome. Aliment Pharmacol Ther 2004; 20(11-12):1253-1269. doi:10.1111/j.1365-2036.2004.02267.x

12. Delvaux M. Role of visceral sensitivity in the pathophysiology of irritable bowel syndrome. Gut 2002; 51(suppl 1):i67-i71. doi:10.1136/gut.51.suppl_1.i67

13. Eshraghian A, Eshraghian $\mathbf{H}$. Interstitial cells of Cajal: a novel hypothesis for the pathophysiology of irritable bowel syndrome. Can J Gastroenterol 2011; 25(5):277-279. doi:10.1155/2011/478370

14. Chey WY, Jin HO, Lee MH, Sun SW, Lee KY. Colonic motility abnormality in patients with irritable bowel syndrome exhibiting abdominal pain and diarrhea. Am J Gastroenterol 2001; 96(5):1499-1506. doi:10.1111/j.1572-0241.2001.03804.x

15. Ford AC, Quigley EM, Lacy BE, et al. Effect of antidepressants and psychological therapies, including hypnotherapy, in irritable bowel syndrome: systematic review and meta-analysis. Am J Gastroenterol 2014; 109(9):1350-1365. doi:10.1038/ajg.2014.148

16. Lackner JM, Jaccard J, Keefer L, et al. Improvement in gastrointestinal symptoms after cognitive behavior therapy for refractory irritable bowel syndrome. Gastroenterology 2018; 155(1):47-57. doi:10.1053/j.gastro.2018.03.063

17. Ford AC, Lacy BE, Harris LA, Quigley EM, Moayyedi P. Effect of antidepressants and psychological therapies in irritable bowel syndrome: an updated systematic review and meta-analysis. Am J Gastroenterol 2019; 114(1):21-39. doi:10.1038/s41395-018-0222-5

18. Annaházi A, Róka R, Rosztóczy A, Wittmann T. Role of antispasmodics in the treatment of irritable bowel syndrome. World J Gastroenterol 2014; 20(20):6031-6043. doi:10.3748/wjg.v20.i20.6031 
19. Kennedy TM, Chalder T, McCrone P, et al. Cognitive behavioural therapy in addition to antispasmodic therapy for irritable bowe syndrome in primary care: randomised controlled trial. Health Technol Assess 2006; 10(19). doi:10.3310/hta10190

20. Siah KT, Wong RK, Ho KY. Melatonin for the treatment of irritable bowel syndrome. World J Gastroenterol 2014; 20(10):2492-2498. doi:10.3748/wjg.v20.i10.2492

21. Cash BD, Epstein MS, Shah SM. A novel delivery system of peppermint oil is an effective therapy for irritable bowel syndrome symptoms. Dig Dis Sci 2016; 61(2):560-571. doi:10.1007/s10620-015-3858-7

22. Crowell MD. Role of serotonin in the pathophysiology of the irritable bowel syndrome. Br J Pharmacol 2004; 141(8):1285-1293. doi:10.1038/sj.bjp.0705762

23. Dunlop SP, Coleman NS, Blackshaw E, et al. Abnormalities of 5-hydroxytryptamine metabolism in irritable bowel syndrome. Clin Gastroenterol Hepatol 2005; 3(4):349-357. doi:10.1016/s1542-3565(04)00726-8

24. Atkinson W, Lockhart S, Whorwell PJ, Keevil B, Houghton LA. AItered 5-hydroxytryptamine signaling in patients with constipationand diarrhea-predominant irritable bowel syndrome. Gastroenterology 2006; 130(1):34-43. doi:10.1053/j.gastro.2005.09.031

25. Ford AC, Brandt LJ, Young C, Chey WD, Foxx-Orenstein AE, Moayyedi P. Efficacy of 5-HT3 antagonists and 5-HT4 agonists in irritable bowel syndrome: systematic review and meta-analysis. Am J Gastroenterol 2009; 104(7):1831-1843. doi:10.1038/ajg.2009.223

26. Miller DP, Alfredson T, Cook SF, Sands BE, Walker AM. Incidence of colonic ischemia, hospitalized complications of constipation, and bowel surgery in relation to use of alosetron hydrochloride. Am J Gastroenterol 2003; 98(5):1117-1122. doi:10.1111/j.1572-0241.2003.07418.x

27. Garsed K, Chernova J, Hastings M, et al. A randomised trial of ondansetron for the treatment of irritable bowel syndrome with diarrhoea. Gut 2014; 63(10):1617-1625. doi:10.1136/gutjnl-2013-305989

28. Lembo AJ, Lacy BE, Zuckerman MJ, et al. Eluxadoline for irritable bowel syndrome with diarrhea. N Engl J Med 2016; 374(3):242-253. doi:10.1056/NEJMoa1505180

29. Cash BD, Lacy BE, Schoenfeld PS, Dove LS, Covington PS. Safety of eluxadoline in patients with irritable bowel syndrome with diarrhea. Am J Gastroenterol 2017; 112(2):365-374. doi:10.1038/ajg.2016.542

30. Barshop K, Staller K. Eluxadoline in irritable bowel syndrome with diarrhea: rationale, evidence and place in therapy. Ther Adv Chronic Dis 2017; 8(11):153-160. doi:10.1177/2040622317714389

31. Zhang FM, Li S, Ding L, et al. Effectiveness of mesalazine to treat irritable bowel syndrome: a meta-analysis. Medicine (Baltimore) 2019; 98(28):e16297. doi:10.1097/MD.0000000000016297

32. Carroccio A, Giannone G, Mansueto P, et al. Duodenal and rectal mucosa inflammation in patients with non-celiac wheat sensitivity. Clin Gastroenterol Hepatol 2019; 17(4):682-690.e3. doi:10.1016/j.cgh.2018.08.043

33. Dunlop SP, Jenkins D, Neal KR, et al. Randomized, double-blind, placebo-controlled trial of prednisolone in post-infectious irritable bowel syndrome. Aliment Pharmacol Ther 2003; 18(1):77-84 doi:10.1046/j.1365-2036.2003.01640.x

34. Huerta C, García Rodríguez LA, Wallander MA, Johansson S. Users of oral steroids are at a reduced risk of developing irritable bowel syndrome. Pharmacoepidemiol Drug Saf 2003; 12(7):583-588. doi:10.1002/pds.836

35. Klooker TK, Braak B, Koopman KE, et al. The mast cell stabiliser ketotifen decreases visceral hypersensitivity and improves intestinal symptoms in patients with irritable bowel syndrome. Gut 2010; 59(9):1213-1221. doi:10.1136/gut.2010.213108

36. Barbara G, Stanghellini V, Cremon C, et al. Aminosalicylates and other anti-inflammatory compounds for irritable bowel syndrome. Dig Dis 2009; 27(suppl 1):115-121. doi:10.1159/000268131

37. Wouters MM, Balemans D, Van Wanrooy S, et al. Histamine receptor H1-mediated sensitization of TRPV1 mediates visceral hypersensitivity and symptoms in patients with irritable bowel syndrome. Gastroenterology 2016; 150(4):875-887.e9. doi:10.1053/j.gastro.2015.12.034

38. Lobo B, Ramos L, Martínez C, et al. Downregulation of mucosal mast cell activation and immune response in diarrhoeairritable bowel syndrome by oral disodium cromoglycate: a pilot study. United European Gastroenterol J 2017; 5(6):887-897. doi:10.1177/2050640617691690

39. Zhang L, Song J, Hou X. Mast cells and irritable bowel syndrome: from the bench to the bedside. J Neurogastroenterol Motil 2016; 22(2):181-192. doi:10.5056/jnm15137

40. Cremon C, Stanghellini V, Barbaro MR, et al. Randomised clinical trial: the analgesic properties of dietary supplementation with palmitoylethanolamide and polydatin in irritable bowel syndrome. Aliment Pharmacol Ther 2017; 45(7):909-922. doi:10.1111/apt.13958

41. Litleskare S, Rortveit G, Eide GE, Hanevik K, Langeland N, Wensaas KA. Prevalence of irritable bowel syndrome and chronic fatigue 10 years after giardia infection. Clin Gastroenterol Hepatol 2018; 16(7):1064-1072.e4. doi:10.1016/j.cgh.2018.01.022

42. Pimentel $\mathbf{M}$, Morales $\mathbf{W}$, Rezaie $\mathbf{A}$, et al. Development and validation of a biomarker for diarrhea-predominant irritable bowel syndrome in human subjects. PLoS One 2015; 10(5):e0126438. doi:10.1371/journal.pone.0126438

43. Talley NJ, Holtmann G, Walker MM, et al. Circulating anti-cytolethal distending toxin $\mathrm{B}$ and anti-vinculin antibodies as biomarkers in community and healthcare populations with functional dyspepsia and irritable bowel syndrome. Clin Transl Gastroenterol 2019; 10(7):e00064. doi:10.14309/ctg.0000000000000064

44. Zhou Q, Verne ML, Fields JZ, et al. Randomised placebo-controlled trial of dietary glutamine supplements for postinfectious irritable bowel syndrome. Gut 2019; 68(6):996-1002. doi:10.1136/gutjnl-2017-315136

45. Ford AC, Gibson PR. Efficacy of glutamine in postinfection IBS. Gut 2019; 68(10):1905-1906. doi:10.1136/gutjnl-2018-317438

46. Atkinson $\mathbf{W}$, Sheldon TA, Shaath $\mathbf{N}$, Whorwell PJ. Food elimination based on IgG antibodies in irritable bowel syndrome: a randomised controlled trial. Gut 2004; 53(10):1459-1464. doi:10.1136/gut.2003.037697

47. Shepherd SJ, Gibson PR. Fructose malabsorption and symptoms of irritable bowel syndrome: guidelines for effective dietary management. J Am Diet Assoc 2006; 106(10):1631-1639. doi:10.1016/j.jada.2006.07.010

48. Halmos EP, Power VA, Shepherd SJ, Gibson PR, Muir JG. A diet low in FODMAPs reduces symptoms of irritable bowel syndrome. Gastroenterology 2014; 146(1):67-75.e5. doi:10.1053/j.gastro.2013.09.046

49. Böhn L, Störsrud S, Liljebo T, et al. Diet low in FODMAPs reduces symptoms of irritable bowel syndrome as well as traditional dietary advice: a randomized controlled trial. Gastroenterology 2015; 149(6):1399-1407.e2. doi:10.1053/j.gastro.2015.07.054

50. Eswaran SL, Chey WD, Han-Markey T, Ball S, Jackson K. A randomized controlled trial comparing the low FODMAP diet vs. modified NICE guidelines in US adults with IBS-D. Am J Gastroenterol 2016; 111(12):1824-1832. doi:10.1038/ajg.2016.434

51. Biesiekierski JR, Newnham ED, Irving PM, et al. Gluten causes gastrointestinal symptoms in subjects without celiac disease: a doubleblind randomized placebo-controlled trial. Am J Gastroenterol 2011; 106(3):508-514. doi:10.1038/ajg.2010.487

52. Biesiekierski JR, Peters SL, Newnham ED, Rosella O, Muir JG, Gibson PR. No effects of gluten in patients with self-reported non-celiac gluten sensitivity after dietary reduction of fermentable, poorly absorbed, short-chain carbohydrates. Gastroenterology 2013; 145(2):320-328.e1-3. doi:10.1053/j.gastro.2013.04.051

53. Simrén M, Barbara G, Flint HJ, et al; Rome Foundation Committee. Intestinal microbiota in functional bowel disorders: a Rome foundation report. Gut 2013; 62(1):159-176. doi:10.1136/gutjnl-2012-302167

54. Saad RJ, Chey WD. Breath testing for small intestinal bacterial overgrowth: maximizing test accuracy. Clin Gastroenterol Hepatol 2014; 12(12):1964-1972. doi:10.1016/j.cgh.2013.09.055

55. Shah ED, Basseri RJ, Chong K, Pimentel M. Abnormal breath testing in IBS: a meta-analysis. Dig Dis Sci 2010; 55(9):2441-2449. 


\section{KURIN AND COOPER}

doi:10.1007/s10620-010-1276-4

56. Posserud I, Stotzer PO, Björnsson ES, Abrahamsson H, Simrén M. Small intestinal bacterial overgrowth in patients with irritable bowel syndrome. Gut 2007; 56(6):802-808. doi:10.1136/gut.2006.108712

57. Bennet SM, Böhn L, Störsrud S, et al. Multivariate modelling of faecal bacterial profiles of patients with IBS predicts responsiveness to a diet low in FODMAPs. Gut 2018; 67(5):872-881. doi:10.1136/gutjnl-2016-313128

58. Hod K, Ringel Y. Probiotics in functional bowel disorders. Best Pract Res Clin Gastroenterol 2016; 30(1):89-97. doi:10.1016/j.bpg.2016.01.003

59. Pimentel M, Lembo A, Chey WD, et al; TARGET Study Group. Rifaximin therapy for patients with irritable bowel syndrome without constipation. N Engl J Med 2011; 364(1):22-32. doi:10.1056/NEJMoa1004409

60. Lembo A, Pimentel M, Rao SS, et al. Repeat treatment with rifaxi$\mathrm{min}$ is safe and effective in patients with diarrhea-predominant irritable bowel syndrome. Gastroenterology 2016; 151(6):1113-1121. doi:10.1053/j.gastro.2016.08.003

61. Sharara Al, Aoun E, Abdul-Baki H, Mounzer R, Sidani S, Elhajj I. A randomized double-blind placebo-controlled trial of rifaximin in patients with abdominal bloating and flatulence. Am J Gastroenterol 2006; 101(2):326-333. doi:10.1111/j.1572-0241.2006.00458.x

62. Johnsen PH, Hilpüsch F, Cavanagh JP, et al. Faecal microbiota transplantation versus placebo for moderate-to-severe irritable bowel syndrome: a double-blind, randomised, placebo-controlled, parallel-group, single-centre trial. Lancet Gastroenterol Hepatol 2018; 3(1):17-24. doi:10.1016/\$2468-1253(17)30338-2

63. El-Salhy M, Hatlebakk JG, Gilja OH, Bråthen Kristoffersen A, Hausken T. Efficacy of faecal microbiota transplantation for patients with irritable bowel syndrome in a randomised, double-blind, placebocontrolled study. Gut 2019. doi:10.1136/gutjnl-2019-319630. Epub ahead of print.

64. Camilleri M. Bile acid diarrhea: prevalence, pathogenesis, and therapy. Gut Liver 2015; 9(3):332-339. doi:10.5009/gnl14397

65. Wedlake L, A'Hern R, Russell D, Thomas K, Walters JR, Andreyev HJ. Systematic review: the prevalence of idiopathic bile acid malabsorption as diagnosed by SeHCAT scanning in patients with diarrhoeapredominant irritable bowel syndrome. Aliment Pharmacol Ther 2009; 30(7):707-717. doi:10.1111/j.1365-2036.2009.04081.x

66. Sauter GH, Münzing W, von Ritter C, Paumgartner G. Bile acid malabsorption as a cause of chronic diarrhea: diagnostic value of 7alpha-hydroxy-4-cholesten-3-one in serum. Dig Dis Sci 1999; 44(1):14-19. doi:10.1023/a:1026681512303

67. Pattni SS, Brydon WG, Dew T, et al. Fibroblast growth factor 19 in patients with bile acid diarrhoea: a prospective comparison of FGF19 serum assay and SeHCAT retention. Aliment Pharmacol Ther 2013; 38(8):967-976. doi:10.1111/apt.12466

68. Vijayvargiya P, Camilleri M, Carlson $\mathbf{P}$, et al. Performance characteristics of serum C4 and FGF19 measurements to exclude the diagnosis of bile acid diarrhoea in IBS-diarrhoea and functional diarrhoea. Aliment Pharmacol Ther 2017; 46(6):581-588. doi:10.1111/apt.14214

69. Odunsi-Shiyanbade ST, Camilleri M, McKinzie S, et al. Effects of chenodeoxycholate and a bile acid sequestrant, colesevelam, on intestinal transit and bowel function. Clin Gastroenterol Hepatol 2010; 8(2):159-165. doi:10.1016/j.cgh.2009.10.020

Address: Michael Kurin, MD, Digestive Health Institute, University Hos pitals Cleveland Medical Center, 11100 Euclid Avenue, Wearn Bldg, Suite 247, Cleveland, OH 44106; michael.kurin@uhhospitals.org 